\title{
TV White Space Standardization and Regulation in Europe
}

\author{
Markus Mueck \\ Infineon Technologies AG \\ Am Campeon 1-12 \\ 85579 Neubiberg, Germany \\ Email: MarkusDominik.Mueck@infineon.com
}

\author{
Dominique Noguet \\ Minatec CEA-LETI \\ 17 , rue des Martyrs \\ 38054 Grenoble Cedex 9, France \\ Email: dominique.noguet@cea.fr
}

\begin{abstract}
Regulators around the world are currently setting rules and requirements that will allow for mass-market access to TV White Spaces. It is expected that the opening of those frequency bands to the consumer market will lead to a dramatic increase of spectrum exploitation efficiency in highly appealing frequency bands: In Europe, the frequency bands between 470 $\mathrm{MHz}$ and $790 \mathrm{MHz}$ are considered and the inherent signal propagation properties are largely superior to those in standard cellular bands, for example, in $900 \mathrm{MHz}$ and $2 \mathrm{GHz}$. This paper will present related use cases for the exploitation of TV White Space as they are currently discussed in the ETSI Reconfigurable Radio Systems Technical Committee. Then, the availability of TV White Spaces in Europe is investigated, followed by an overview of recent regulatory activities on the European Commission level. In particular, expectations related to a new revision of the European R\&TTE Directive are presented which represents the basic regulatory framework in Europe.
\end{abstract}

Keywords- Cognitive Radio (CR), ETSI, Software Defined Radio (SDR)

\section{INTRODUCTION}

The idea of allowing for an opportunistic usage of TV White Spaces emerged from the broader Cognitive Radio (CR) as proposed by [1]. The CR principles suggest that any types of network devices (on the Mobile Device (MD) and Infrastructure side) are continuously observing the (radio, business, etc.) context and adapt their behavior in the most optimum way possible. Typically, MDs target a configuration that allows to optimize the MD configuration from their perspective - typically targeting a minimum level of power consumption, low subscription costs, etc. From the network side, the overall optimization of network efficiency is targeted as well as the maximization of related revenues. Recent progress in this field paved the way for exploiting frequency bands opportunistically (i.e., access by secondary devices is possible whenever non harmful interference with the incumbent systems is guaranteed). In particular TV Whites (TVWS) were chosen to be the first playground for deploying first generations of the technology. Besides the emergence of CR, TV switch over to full digital broadcast service created new spectrum opportunities due to higher spectrum efficiency compared to analog services. As discussed in [2], the propagation properties are appealing in particular for shortrange and cellular applications due to the superior signal propagation properties compared, for example, to traditional cellular systems operating in $900 \mathrm{MHz}$ and $2 \mathrm{GHz}$ bands or short range systems operating the the $2.4 \mathrm{GHz}$ or $5 \mathrm{GHz}$ bands. In particular, this approach allows for [2]

i) Lighter infrastructure deployment through larger cell sizes;

ii) Increased spectral efficiency through reduced propagation loss;

iii) Increased spectral efficiency through extended macro diversity.

At the same time, TVWS usage by White Space Devices (WSD) requires an adequate protection of primary/incumbent users. In the US, FCC requests the introduction of a data-base [3] that contains information from primary/incumbent users on future intended usage of TVWS over time and space. In the UK, OFCOM seems to undertake the same approach [4]. In Europe, CEPT WG SE43 currently finalized a report [5] that suggests an implementation combining a data-base oriented approach with MD based sensing.

A vision on a corresponding network architecture has been recently proposed in [6] using a combination of distributed and centralized data-bases. It is also obvious that a multitude of standards are currently under elaboration for operation in TVWS in various committees, including ETSI, IEEE, ECMA, etc. An efficient interoperation between the WSD users is expected to require access to related context information - this can for example be provided by a Cognitive Pilot Channel (CPC) mechanism as discussed in [7,8].

In the sequel of this paper, the focus will be on the current status of TVWS standardization in ETSI, which is carried out in the ETSI Reconfigurable Radio Systems (RRS) Technical Committee (TC). It should be noted that PMSE (Programme Making and Special Events) related work for operation in TVWS is currently performed within ETSI ERM STF386 [9], which is out of scope of this paper. Section II will discuss the actual availability of TVWS in Europe, followed by an overview on related Use Cases and Scenarios in Section III as they are currently discussed in ETSI RRS. Section IV comments regulatory activities in Europe in particular related to CEPT WG SE43 and activities related to the current revision of the R\&TTE Directive. Section V finally gives a conclusion. 


\section{AVAILABILITY OF TV White SPACES IN EUROPE}

The availability of TVWS has been studied in the US, among others, by Spectrum Bridge [10]. Since Europe is more densely populated over large areas compared to the US, it is appropriate to wonder whether TVWS are actually available in Europe. Besides, Europe is fragmented by many country borders belonging to different regulation agencies, requiring careful harmonization across borders, which makes the role of CEPT crucial. In particular, Fig. 1 illustrates the DVB-T plan entries of a given channel (channel 21) in Europe and bordering countries over space.

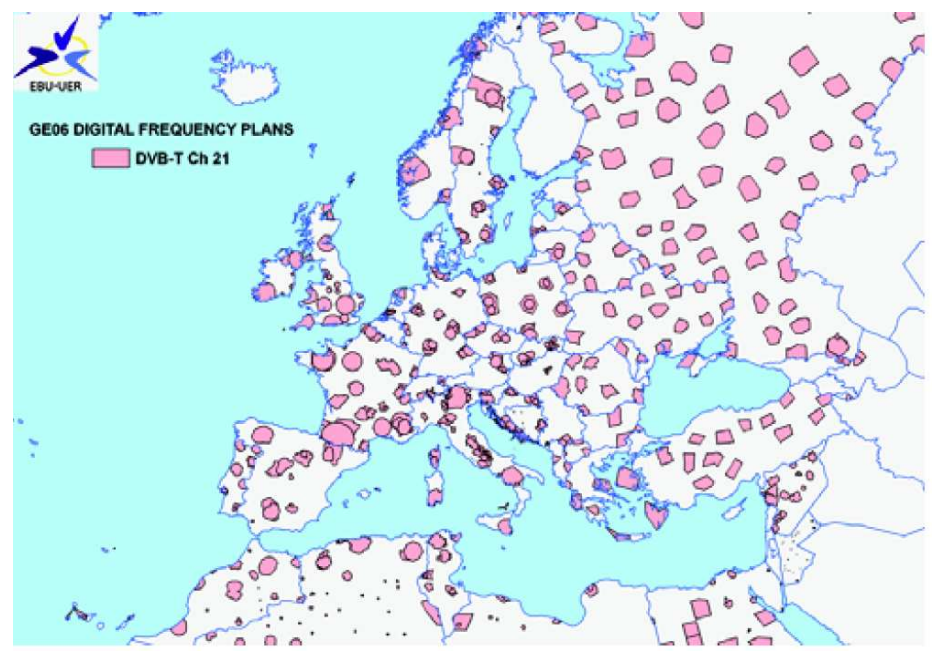

Figure 1: Study results on DVB-T plan entries of Channel 21 in Europe [11].

As it can be expected, TV broadcasters of a densely populated countries exploit TV channels in particular in the major cities. However, a considerably portion of the countries can be identified as "White Spaces" without any usage of the related frequency band by broadcasters. In those areas in particular, the usage of the related bands by secondary spectrum access approaches can be envisaged. In the UK, this border issues are less problematic due to obvious geographical situation, and OFCOM was one of the first regulators to consider WSD in TV bands. OFCOM launched Digital Dividend Reviews as early as 2005. The latest statements related to license exempt operation can be found in [12]. One key concern is to evaluate the expected amount of spectrum available to secondary TVWS devices. Both [5] and [12] show that the answer varies significantly according to the scenarios. One example is illustrated in Fig. 2, which considered, based on various assumptions: Conservative, Optimistic and Adjacents free [12].

Within their limited scope, those studies show that the availability of TVWS in Europe is expected to be less abundant as it is in other areas, such as the US, etc. In particular, the potential usage of WSD in major cities in Europe is still under study. Outside the major cities, however, current results show that the WSD may play a major role for broadband wireless communication.

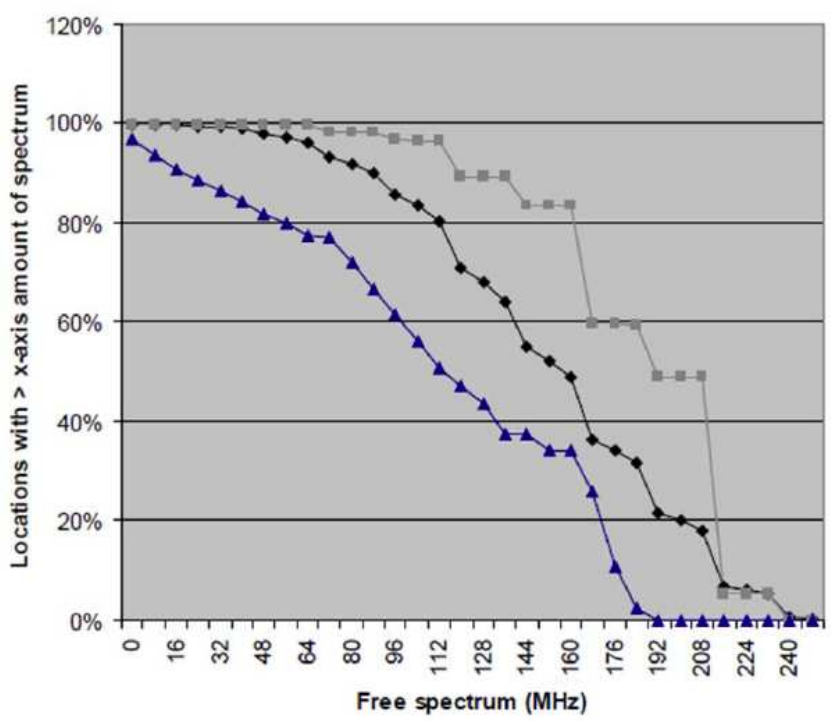

Figure 2: Study results on aggregate spectrum availability [12].

A further issue to be considered is the protection of PMSE devices, such as wireless microphones, operating in TVWS bands as defined by current regulatory rules. In particular, within a given distance of PMSE devices, the corresponding frequency bands cannot by reused by other WSD - this is expected to further limit their possible use in major European cities. PMSE are used on a temporary basis (compared to TV broadcasts) and are difficult to detect because of their low power and modulation scheme. Although safe harbor channels (one or more dedicated channels) are considered for these devices (e.g. in [5]), it is expected that big events will require more spectrum on a temporary ground. Thus the secondary usage by WSD is expected to be far more dynamic than what imagined when considering TV broadcasts only. Mobility is another factor that will make TVWS operation very dynamic as WSD will have to handover channels as they move along.

\section{Use CASES For TV White SPACE USAGE AS DISCUSSED IN ETSI RRS}

The ETSI RRS Technical Committee [13] is the center of competence within ETSI for CR and SDR technology. In particular, a cognitive radio vision has been published in [14] and a number of working items are currently dealing with TVWS related standardization, focusing on both cellular and short range type of applications. In the sequel, key Use Cases and Scenarios are presented as they are currently under discussion within ETSI RRS [15].

1) Use Case: Mid-/long range wireless access over White Space frequency bands

Internet access is provided from a base station to the end users by utilizing White Space frequency bands over ranges similar to today's cellular systems, e.g. in the range of $0-10 \mathrm{~km}$. 
This Use Case is considered in further detail in the following Scenarios:

\section{1) Mid-/long range, no mobility}

In this scenario, wireless access is provided from a base station towards fixed devices, e.g. a fixed mounted home base station/access point. The geo-location from both the base station as well as from the fixed device are well-known.

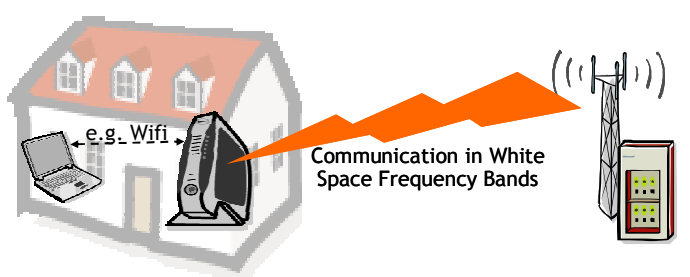

Figure 3: Mid-/long Range Wireless Access, no Mobility.

1.2) Mid-/long range, low mobility

In this scenario, wireless access is provided from a base station towards mobile devices where the users have low mobility, e.g. they are staying at their location or walking. In that respect, sensing results for primary users retrieved for the current location are not getting invalid due to the mobility of the user. The geo-location from the base station is wellknown. The geo-location from the mobile device must be determined during operation, e.g. via GPS or cellular positioning systems.

\section{3) Mid-/long range, high mobility}

In this scenario, wireless access is provided from a base station towards mobile devices and the mobile devices may move fast, e.g. because a user is in a car or a train. In that respect, sensing results for primary users retrieved for the current location may get invalid quickly due to the mobility of the user. Thus, this use case sets high constraints for the detection of primary users and it can be questioned if high mobility will be supported in TV White Spaces at all.

\section{4) Network centric management of TV White Spaces}

We consider here below a network centric solution in regards of allocating available TVWS for the user terminal to get connectivity. In this scenario, available TVWS frequency band is considered based on location rather than in time, it is assumed that TVWS would be largely available in rural area and in time. Once the terminal accesses the network it can be left under the control of the network, high layer signaling can be used for this purpose e.g., handover command to hand-off to a new frequency or system broadcast messages can be used to notify terminals about change of the frequency.

2) Use Case: Short range wireless access over White Space frequency bands

Internet access is provided via short range wireless communication (e.g. in the range of $0-50 \mathrm{~m}$ ) from an access point or base station to the end users by utilizing White Space frequency bands.

This Use Case is considered in further detail in the following Scenarios:

\section{1) Uncoordinated networks}

In this case multiple, uncoordinated networks access White Space frequency bands. This can happen e.g. in an apartment house where residents have their own local area access points operating in White Space frequency band.

In order to work properly, this case requires effective coexistence mechanisms for White Space frequency access.

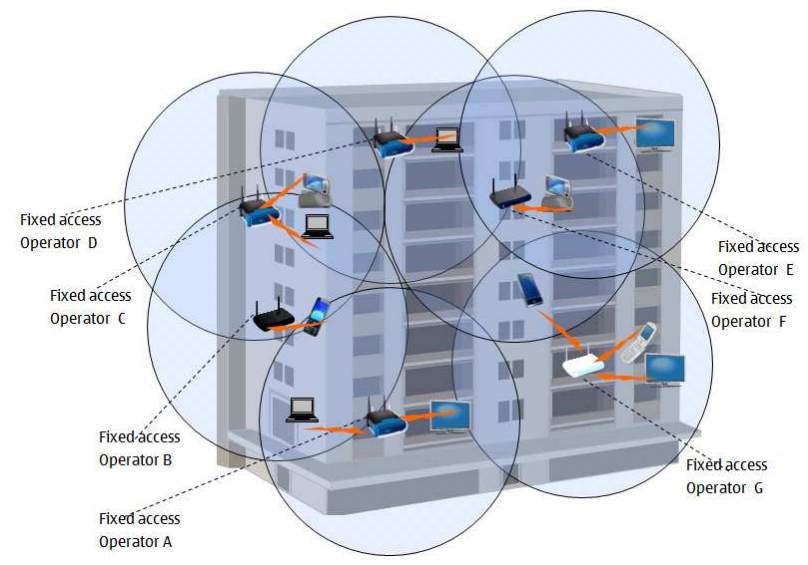

Figure 4: Short Range Wireless Access, Uncoordinated Networks.

\section{2) Coordinated networks}

In this case the White Space networks in the proximity are operated in coordinated manner by a White Space operator. Examples of this kind of usage can be small scale corporate networks, networks for academic institutions etc.

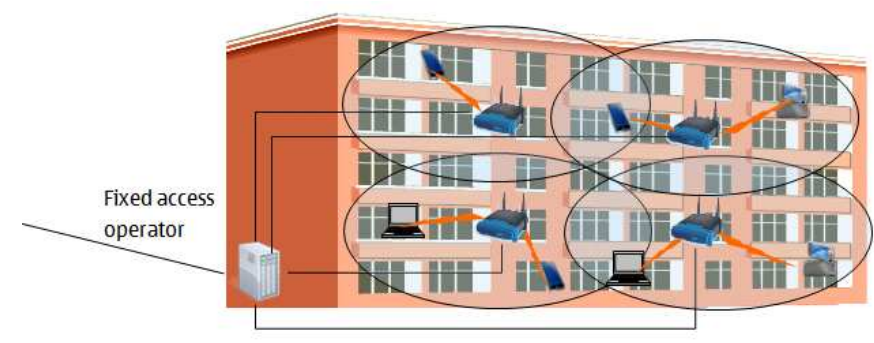

Figure 5: Short Range Wireless Access, Coordinated Networks.

2.3) Hybrid of uncoordinated and coordinated networks In this case networks of the previous two scenarios are overlapping, i.e. the overall deployment consists of both the uncoordinated and coordinated networks. Examples where 
such situations could arise are campus areas, shopping malls etc. In order to work properly, effective coexistence methods need to be in place for this scenario case.

\section{3) Use Case:Ad-hoc networking over White Space frequency} bands

In this use case the devices (user devices and other devices like access points) communicate with each other to share information, to run joint applications or services, or to execute other similar tasks. The communication happens by forming an ad hoc network operating on White Space frequency band. There can be two or more devices in the ad hoc network formed.

\section{1) Device-to-device connectivity}

In this case two devices (which can be similar or different) connect in peer-to-peer manner to exchange information between each other. The information can be e.g. multimedia content, or control information like measurement results shared between the devices. The devices can be similar from their capabilities (like two mobile devices), or then completely different (like a mobile device and external printer or display).

\section{2) Ad-hoc networking}

In this case the devices form an ad hoc network to communicate and collaborate with devices in the neighborhood. As an example the devices can be operating a localized social networking service (possibly maintained by a service provider)

\section{4) Use Case:Opportunistic Access to TVWS by Cellular} Systems during Absence of Primary User

In this use case, TVWS slots are only available sporadically for secondary users, such as for example multi-mode user terminals being able to operate, among other systems, cellular systems in licensed and unlicensed spectrum. The supported unlicensed spectrum is assumed to include TVWS, i.e. the 470-790 MHz range in Europe/Region 1.

The time-limited switch of a Base Station to operate in unlicensed spectrum (in particular TVWS) is leading to a number of advantages which are further detailed in the Scenario descriptions detailed in the sequel.

\section{1) Lighter infrastructure deployment through larger cell} sizes

Due to the improved propagation characteristics in the TVWS bands compared to typical licensed bands, a large cell size is chosen which will lead to a lighter infrastructure deployment and thus to an overall reduced CAPEX/OPEX.

\section{2) Increased spectral efficiency through reduced} propagation loss

Due to the improved propagation characteristics in the TVWS bands compared to typical other licensed bands, a possible deployment choice is to keep a cell size as it is the case for the licensed band deployment. The following advantages are observed:

i) Due to the improved propagation characteristics in the TVWS bands, a higher QoS is achieved within the given cell. However, those propagation characteristics may also increase interference issues which require an adequate handling (e.g. suitable frequency reuse-factor for TVWS, power management, etc.);

ii) Due to the improved propagation characteristics in the TVWS bands, an identical QoS is achieved within the given cell at a lower RBS/MD output power level. The inherent power consumption can be reduced;

iii) A hybrid solution of item i) and ii) is possible, i.e. a moderate reduction of the RBS output power levels combined with a moderate improvement of the QoS.

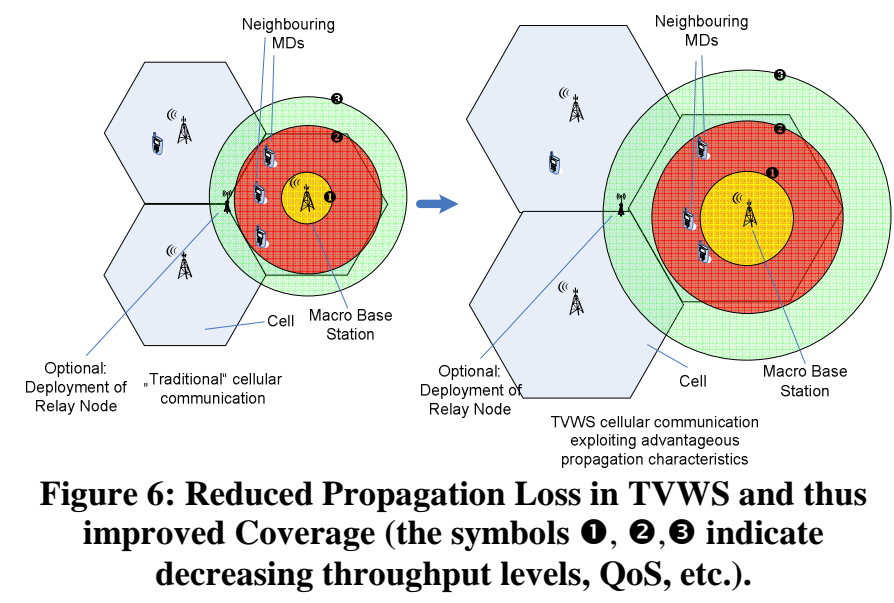

4.3) Increased spectral efficiency through extended macro diversity

Due to the improved propagation characteristics in the TVWS bands compared to typical other licensed bands, a possible deployment choice is to keep a cell size (or to increase it only slightly) as it is the case for the licensed band deployment. Then, joint operation of neighboring RBS can be exploited in order to achieve a higher Macro-Diversity gain in the UL (multiple RBS are decoding jointly the received signals) or in the DL (multiple RBS are contributing to jointly optimized transmission).

\section{REGULATORY ACTIVITIES IN EUROPE RELATED TO COGNITIVE RADIO AND TV WHITE SPACES}

On the regulatory field, possible European approaches for operating secondary TV White Space devices is under study by national regulators and in CEPT WG SE43. A corresponding report [5] is currently about to be finalized. Whilst [5] considered three cognitive techniques (sensing, geo-location database and beacon) at the start of the study, most of the efforts were finally devoted to the assessment of the appropriateness of the sensing and geo-location techniques to provide protection to the incumbent radio services. The following aspects were considered: 
i) Protection of the broadcasting service;

ii) Protection of PMSE;

iii) Protection of Radio Astronomy (RAS) in the 608-614 $\mathrm{MHz}$

iv) Protection of aeronautical radionavigation (ARNS) in the 645-790 MHz band;

v) Protection of Mobile/Fixed services in bands adjacent to the band 470-790 MHz;

vi) Definition of the requirements for the geo-location database approach;

vii) Assessment of the consequential spectrum potentially available for WSD.

It should also be noted that the European R\&TTE Directive, the basic regulatory framework for wireless communication, is currently under revision. It is expected that the new version will include mechanisms that allow for operation of SDR capable MDs in the future - those devices are expected to trigger the development of CR based features that guarantee an efficient exploitation of frequency bands such as the TV WS.

As the study went along, it came clearer than geo-location will play the main role in defining the channels that must be free of WSD transmissions. This strategy is established to guarantee interference free operation of incumbents but is not sufficient to design White Space networks so that they benefit from the best of the available spectrum. Recent research, (e.g. in the QoSMOS project [16]) aims at providing technical solution to this issue while considering regulatory regimes carefully. In QoSMOS, spectrum management (SM) and resource management (RM) are separated entities working on different time scales to respectively create spectrum portfolios which contain all regulation constraints for each opportunities and additional information from the network, and exploit this portfolio to allocate spectrum access using QoS based rules. In this context, it was identified in [17] that spectrum sensing (which may not be strictly needed for incumbent protection) is seen as an efficient mean to optimize WS opportunistic usage optimization.

\section{CONCLUSIONS}

This paper has given an overview on the European framework related to the efficient exploitation of TV WS. In particular, it was shown that TV WS are expected to be available for secondary spectrum access in particular outside of the major cities. Use Cases as discussed in ETSI RRS are outlined, showing that both cellular and short-range applications are envisaged for TV WS access. Finally, an assessment on the regulatory framework is given. In particular, an expected change in the European regulatory framework is outlined (revision of the R\&TTE Directive) which is expected to lead to further opportunities in exploiting White Spaces opportunistically. For the time being, the focus is clearly on TV WS in the $470 \mathrm{MHz}$ to $790 \mathrm{MHz}$ range - for the future though, the authors definitely expect that further bands will be considered as potential candidates for opportunistic access, representing though extended White Spaces. Regulation rules are set to prevent harmful interference to incumbents but do not provide insights to operate the available spectrum efficiently. Current research initiatives are tackling this objective.

\section{ACKNOWLEDGEMENT}

The research leading to these results was derived from the European Community's Seventh Framework Programme (FP7) under Grant Agreement number 248454 (QoSMOS)

\section{References}

[1] J. Mitola III, Cognitive Radio, Ph.D. thesis, KTH, Stockholm, Sweden, 2000

[2] Opportunistic relaying for Cognitive Radio enhanced cellular networks: Infrastructure and initial results, Mueck, Markus Dominik; Di Renzo, Marco; Debbah, Merouane; Wireless Pervasive Computing (ISWPC), 2010 5th IEEE International Symposium on, 2010, Page(s): 556 - 561

[3] http://www.fcc.gov/oet/cognitiveradio

[4] William Webb "Cognitive access to the interleaved channels: Update and next steps" The IET seminar on Cognitive Radio Communication, London, Oct. 2010

[5] CEPT WG SE43, Draft Report ECC 159, "Technical and operational requirements for the possible operation of cognitive radio systems in the "white spaces" of the frequency band 470-790 MHz", available at http://www.ero.dk

[6] Combination of Centralized \& Decentralized Database and Terminalbased Spectrum Sensing for Secondary Spectrum Access, Markus Mueck, Marco Di Renzo, Mérouane Debbah and Tobias Renk, IEEE International Conference on Wireless Information Technology and Systems (ICWITS), Hawaii, USA, 2010

[7] Demand driven evolution of the Cognitive Pilot Channel; Mueck, M.; Haustein, T.; Cognitive Radio Oriented Wireless Networks \& Communications (CROWNCOM), 2010 Proceedings of the Fifth International Conference on , Publication Year: 2010, Page(s): 1 - 5

[8] A Local Cognitive Pilot Channel (LCPC) for neighbourhood discovery, relaying and cluster based local cognitive information management; Mueck, M.; Hayar, A.; Cognitive Radio Oriented Wireless Networks \& Communications (CROWNCOM), 2010 Proceedings of the Fifth International Conference on; Publication Year: 2010 , Page(s): $1-5$

[9] Terms of Reference for Specialist Task Force STF 386 (PMSE) - Cognitive Radio; ERM / TG17 WP3: "Methods, parameters and test procedures for cognitive interference mitigation techniques for use by PMSE devices (Programme Making and Special Events)", available at http://portal.etsi.org

[10] Spectrum Bridge, Dec 2009, http://www.showmywhitespace.com

[11] T. OLeary, E. Puigrefagut, W. Sami, "GE06 - overview of the second session (RRC-06) and the main features for broadcasters", EBU Technical Review, October 2006, 20 pp.

[12] OFCOM, "Digital dividend: cognitive access ",Statement on licence exempting cognitive devices using interleaved spectrum, July 2009, Available: $\quad$ http://www.ofcom.org.uk/consult/condocs/cognitive/ statement/statement.pdf

[13] ETSI Reconfigurable Radio Systems Technical Committee (ETSI RRS TC), information available at http://portal.etsi.org

[14] ETSI TR 102 802: "Reconfigurable Radio Systems (RRS); Cognitive Radio System Concept", 2009, available at http://www.etsi.org

[15] ETSI TR 102 907, Reconfigurable Radio Systems (RRS); Use cases for Operation in White Space Frequency Bands, to be published

[16] QoSMOS: Quality of Service and MObility driven cognitive radio Systems, European FP7 ICT Integrated Project, http://www.ictqosmos.eu

[17] QoSMOS deliverable D3.2 Reference Protocol Stack for QoSMOS Radio Environment Mapping and Sensing, http://www.ict-qosmos.eu, Oct 2010. 